\title{
El oxígeno suplementario no fue útil en personas con enfermedad pulmonar obstructiva crónica con desaturación moderada
}

\author{
Supplemental oxygen was not useful in people with chronic obstructive pulmonary disease with moderate desaturation
}

Long-Term Oxygen Treatment Trial Research Group y col. N Engl J Med 2016;375:1617-27

\section{Objetivo}

Evaluar si el uso de oxígeno suplementario disminuye la mortalidad y la incidencia de hospitalizaciones en pacientes con enfermedad pulmonar obstructiva crónica (EPOC) y desaturación moderada en reposo o inducida por el ejercicio.

\section{Diseño, lugar y pacientes}

Ensayo clínico aleatorizado de grupos paralelos realizado en 47 centros en EE.UU. Fueron incluidos 738 pacientes estables con desaturación moderada en reposo -SpO2 89 a 93\%- o inducida por el ejercicio -mayor a $80 \%$ por más de cinco minutos y menor a $90 \%$ por más de diez segundos durante el test de marcha de seis minutos (TM6M).

\section{Intervención y medición de resultados principales}

Fueron aleatorizados en dos grupos en forma no enmascarada. A los pacientes del grupo intervención se les prescribió 24 horas de oxígeno suplementario si su $\mathrm{SpO} 2$ en reposo estaba entre 89 y $93 \%$, y oxígeno solamente durante el sueño y el ejercicio si desaturaban sólo durante el ejercicio. El grupo control no recibió oxígeno suplementario. El resultado principal fue el tiempo hasta la primera hospitalización o la muerte, y los secundarios, la incidencia de exacerbación de la EPOC, la adherencia al oxígeno suplementario, el desarrollo de desaturación severa, la distancia caminada en el TM6M, la calidad de vida, y los niveles de ansiedad y depresión. Los datos fueron analizados por intención a tratar.

\section{Resultados principales}

Los pacientes fueron seguidos una mediana de 18,4 meses. El tiempo hasta el evento no fue significativamente diferente entre los grupos. Tampoco se encontraron diferencias en las variables secundarias. Los resultados principales se muestran en la tabla 1.

Tabla 1. Comparación de la incidencia de muerte y de al menos una hospitalización en pacientes con enfermedad pulmonar obstructiva crónica estable con desaturación moderada en reposo o durante el ejercicio.

\begin{tabular}{|c|c|c|c|c|}
\hline \multirow{2}{*}{ Eventos } & \multicolumn{2}{|c|}{ Incidencia por cada 100 personas/año } & \multirow{2}{*}{$\begin{array}{l}\text { Hazard ratio } \\
\text { (IC 95\%) }\end{array}$} & \multirow{2}{*}{$p$} \\
\hline & Control $(n=370)$ & Intervención ( $n=368$ ) & & \\
\hline Muerte & $5,7(73)$ & $5,2(66)$ & $0,90(0,64$ a 1,25$)$ & 0,53 \\
\hline Primera Hospitalización & $34,5(237)$ & $31,6(229)$ & $0,92(0,77$ a 1,10$)$ & 0,37 \\
\hline
\end{tabular}

IC 95\%: Intervalo de confianza del 95\%.

\section{Conclusiones}

En pacientes con EPOC estable y desaturación moderada en reposo o inducida por el ejercicio, el oxígeno suplementario a largo plazo no disminuye la mortalidad ni la probabilidad de hospitalización.
Fuente de financiamiento: National Heart, Lung, and Blood Institute, $\mathrm{Na}-$ tional Institutes of Health and Department of Health and Human Services, Centers for Medicare and Medicaid Services, Department of Health and Human Services.

\section{Comentario}

La disminución de las exacerbaciones y la mortalidad son los objetivos más importantes del tratamiento de pacientes con EPOC. Si bien la oxigenoterapia continua ya había demostrado mejorar la sobrevida en pacientes con desaturación severa ${ }^{1,2}$, en el estudio que hemos resumido -el de mayor tamaño muestral de los publicados hasta la actualidad-, no se observaron los mismos resultados en pacientes menos graves.

La ausencia de beneficios de la utilización de oxígeno en el subgrupo de pacientes con desaturación inducida con el ejercicio podría deberse a que estas personas pasan menos del $6 \%$ de su tiempo caminando ${ }^{3}$. Vale destacar que este estudio no evaluó dos variables importantes que hacen a la calidad de vida de esta población: la disnea y la actividad física diaria (AFD). Además, el TM6M, no es el más sensible para detectar cambios con una intervención como la evaluada ${ }^{4}$; por lo que quizás estos resultados no concuerdan con los de Haidl y col. ${ }^{5}$, que documentó en esta subpoblación que utilizar oxígeno suplementario disminuye la disnea y mejora la tolerancia al ejercicio utilizando una prueba a carga constante. Por otro lado, una revisión Cochrane ${ }^{6}$ reciente comunicó -con moderada calidad de evidencia- que esta intervención se asocia a un alivio de la disnea en pacientes con hipoxemia moderada e incluso normoxemia durante el ejercicio.

Se ha demostrado que el oxígeno suplementario disminuye la frecuencia respiratoria y la hiperinsuflación dinámica durante el ejercicio, permitiendo mayor tolerancia a la rehabilitación pulmonar. Por este motivo, su uso podría considerarse en algunos individuos seleccionados ${ }^{7}$, combinado con estrategias para aumentar la AFD (como el tele-coaching) y valorando su impacto sobre la mortalidad y las exacerbaciones, dos resultados que mejoran con el aumento de AFD.

\section{Conclusiones de la comentadora}

El oxígeno suplementario en pacientes con EPOC y desaturación moderada no debe indicarse en forma continua, pero debe considerarse su uso durante el ejercicio y debería evaluarse de forma individualizada la mejoría de la disnea y el aumento de la AFD.

Silvina Dell'Era [Hospital Italiano de Buenos Aires. silvina.dellera@hospitalitaliano.org.ar]

Dell'Era S. El oxígeno suplementario no fue útil en personas con enfermedad pulmonar obstructiva crónica con desaturación moderada. Evid Act Pract Ambul. 2018;21(1):31. Comentado de: Long-Term Oxygen Treatment Trial Research Group y col. A Randomized Trial of Long-Term Oxygen for COPD with Moderate Desaturation. N Engl J Med. 2016 Oct 27;375(17):1617-1627. PMID:27783918

Referencias Bibliográficas

1- Continuous or nocturnal oxygen therapy in hypoxemic chronic obstructive lung disease: a clinical trial. Nocturnal Oxygen Therapy Trial Group. Ann Intern Med. 1980 Sep;93(3):391-8

2- Long term domiciliary oxygen therapy in chronic hypoxic cor pulmonale complicating chronic bronchitis and emphysema. Report of the Medical Research Council Working Party. Lancet. 1981 Mar 28;1(8222):681-6.

3- Pitta F y col. Characteristics of physical activities in daily life in chronic obstructive pulmonary disease. Am J Respir Crit Care Med. 2005 May 1;171(9):972-7.

4- Puente-Maestu L y col. Use of exercise testing in the evaluation of interventional efficacy: an official ERS statement. Eur Respir J 2016; 47: 429-460.

5-Haidl P y col. Long-term oxygen therapy stops the natural decline of endurance in COPD patients with reversible hypercapnia. Respiration. 2004 Jul-Aug;71(4):342-7.

6 - Ekström M y col. Oxygen for breathlessness in patients with chronic obstructive pulmonary disease who do not qualify for home oxygen therapy. Cochrane Database Syst Rev. 2016 Nov 25:11:CD006429.

7- Somfay A y col. Dose-response effect of oxygen on hyperinflation and exercise endurance in non hypoxaemic COPD patients. Eur Respir J. 2001 Jul;18(1):77-84. 